\title{
MODÈLE D'UNE CAGE A DIGESTIBILITÉ POUR PETITS RUMINANTS
}

PAR

\section{R. BOCCARD et J. M. BOISSAU}

Station de Recherches sur 1'Élevage, C. N. R. Z., Jouy-en-Josas (Seine-et-Oise).

La détermination classique de la digestibilité des aliments nécessite la connaissance précise des ingesta et des excréta de chacun des individus en expérience. Dans la pratique des mesures, la collection totale et séparée des urines et des fèces constitue la difficulté essentielle.

L'utilisation de mâles permet d'obtenir de bons résultats quand on les place dans des cages conçues pour obtenir séparément fèces et urines. La séparation est obtenue soit par un système de grille et d'entonnoir (Sotola I927, Hogdson et coll. 1935, Bratzlekr I95i, Nelson et col. I954), soit en équipant les animaux d'un système de harnais, de sac et d'entonnoir (ForBes et col. I937, BURKIT'T I95I, RAYMOND et col. I953, DICK et MULES I955).

Mais certaines expériences de métabolisme, la mesure des bilans, à des périodes précises de la vie des animaux, obligent parfois à utiliser des femelles. Avec celles-ci la séparation sans contamination des urines et des fèces présente plus de difficultés.

La solution pourrait résider dans l'utilisation d'un cathéter maintenu dans l'urètre par un harnais (BASSEr I952), mais cet appareil ne peut rester en place que quelques jours.Comme les périodes de mesure doivent parfois être longues, il faut utiliser des procédés de séparation ne perturbant qu'au minimum la vie de l'animal.

BRATZLER (I95I) a présenté une cage à digestibilité permettant 1'utilisation des mâles et des femelles. La séparation des fèces et des urines y est obtenue par poche métallique et plans inclinés.

Nous présentons ici un modèle de cage reposant sur le même principe, mais de conception plus simple, rendant plus aisé l'accès des grilles et du plan incliné.

Io cages de ce modèle sont en service depuis un an dans notre centre et ont été utilisées aussi bien pour des veaux que pour des brebis. 


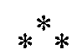

La cage à digestibilité, destinée à des expériences sur moutons, brebis et veaux, est constituée par un bâti en bois sur lequel les différents organes sont montés.

Seuls ces organes appellent quelques détails (fig. I, 2, 3 cotes en mm.)

\section{I. - Contention de l'animal}

Dans les premières cages utilisées pour les expériences de digestibilité (SoTola 1927, Hodgson et coll. I935), les animanx pouvaient se mouvoir

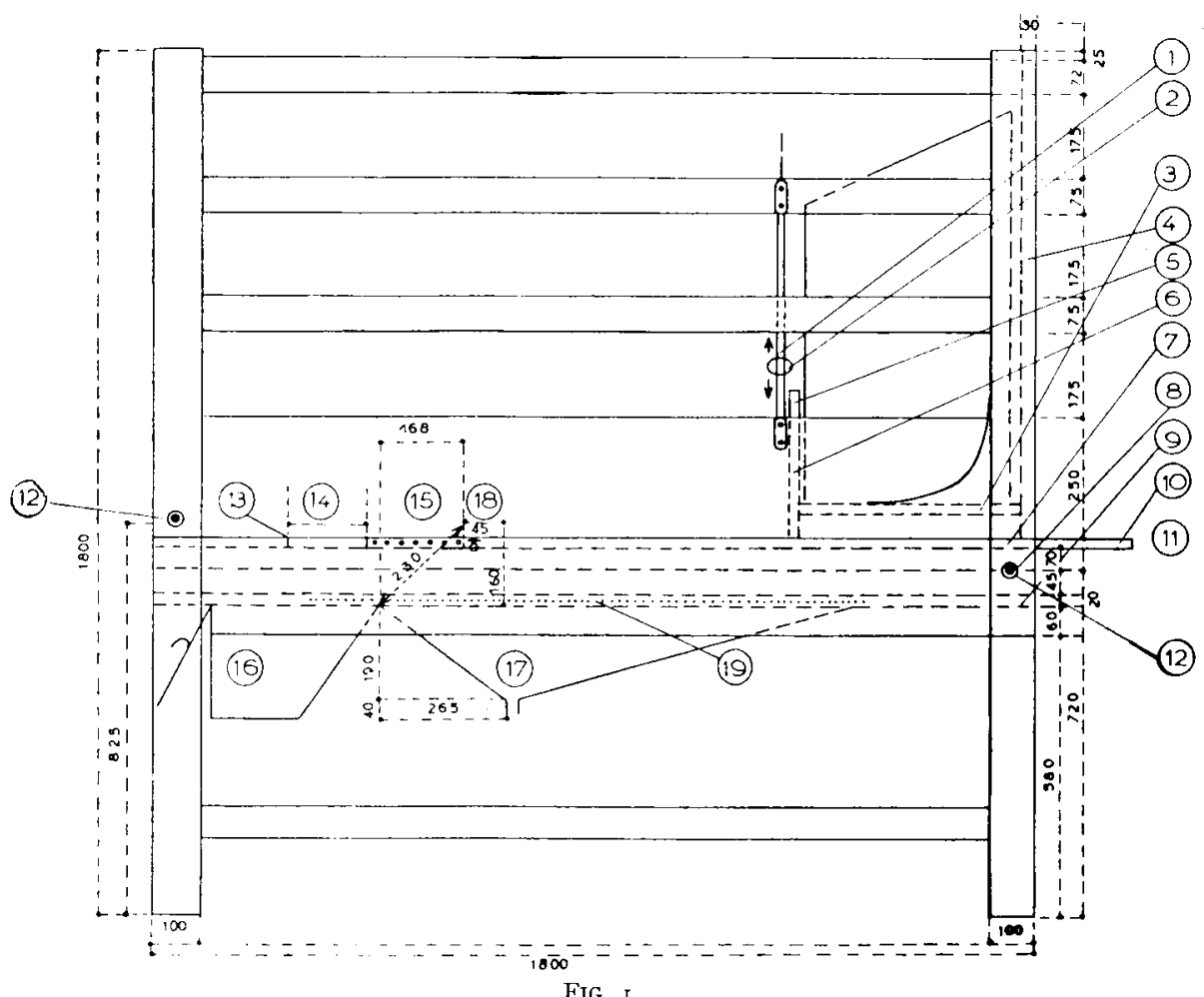

librement sur un espace relativement large. L'utilisation de femelles et l'obtention de fèces aussi peu souillées d'urine que possible, oblige à maintenir l'animal dans une position donnée.

Dans notre cage 1'animal est équipé d'un collier muni de deux cordelettes, chacune d'elles est terminée par un anneau (2) qui coulisse dans deux barres verticales ( $\mathrm{r}$ ) fixées à l'intérieur des parois latérales du bâti de bois. 
Ce système, peu coûteux, permet de limiter le déplacement longitudinal de l'animal sans l'empêcher de se coucher.

Ce mouvement est, de plus, limité à l'avant par l'auge à fourrage ou par une planche de maintien amovible (5) lorsque les aliments sont présentés dans des seaux. Vers l'arrière une saillie du plancher, obtenue par la grille inclinée séparatrice de fèces (I8) ou la partie libre (I4) limite le recul de l'animal.

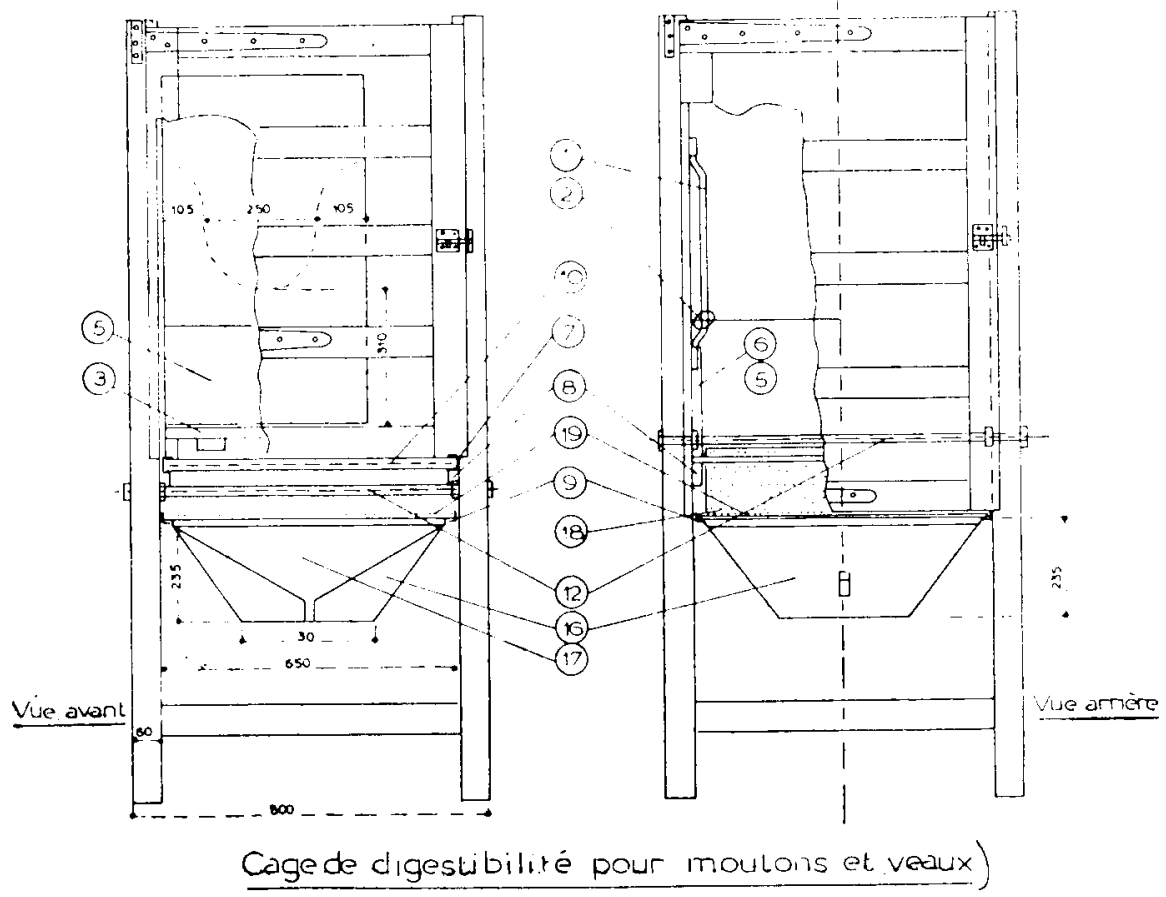

FIG. 2.

Éventuellement, pour de petits animaux, des bas flancs accrochés sur les côtés de bois permettent de diminuer la place disponible et de limiter les déplacements latéraux.

\section{2. - Alimentation}

L'affouragement se fait dans des auges en tôle glavanisée, dont la face avant est percée d'une ouverture en U.

Le fond de l'auge est ceintré pour faciliter la descente du fourrage vers l'animal.

La récupération des refus éventuels se fait aisément en basculant l'auge autour de charnières fixées sur le bas de la face arrière.

Si la ration est constituée d'aggloméré, il est possible, au lieu et place 
de l'auge à fourrage, de disposer des seaux ou bacs sur la plateforme d'alimentation (3).

Il faut dans ce cas mettre en place la planche de maintien (5).

\section{3. - Abreuvement}

Avec l'auge à fourrage, l'eau est mise à la disposition de l'animal dans de petits bacs, placés à l'extérieur de l'auge sur la plateforme (3) ou accrochés au bâti en bois.

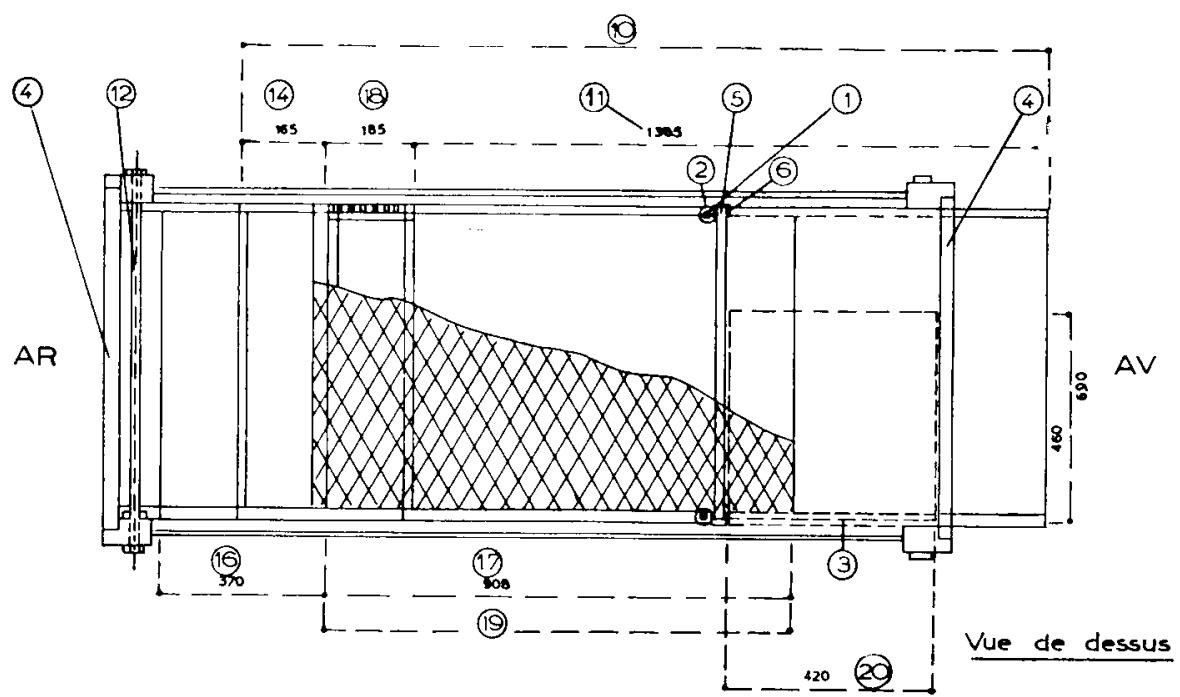

Fig. 3. - Cage de digestibilité pour moutons brebis et veaux.

(I) Barres d'attache pour le coulissement vertical dis anneaux de fixation des cordelettes. (2) Anneaux reliés par deux cordes au collier. - (3) Plate-forme d'alimentation. - (4) Portes. (5) Planche de maintien avant amovible (glissière vers le haut). - (6) Glissière de (5) tôle pliée en U. - (7) Glissière de plancher (mêmes caractéristiques que (6)). - (8) Tasseau de support de (7). - (9) Glissière de l'entonnoir à urine et du bac à fèces (fer cornière $20 \times 20 \mathrm{~mm}$ ). - (Io) Plancher réglable de $I_{73} \times 65 \mathrm{~cm}$. -... (I I) Partie utile maximum du plancher (longueur $\mathrm{I} 38,5 \mathrm{~cm}$ ). - ( ( 2) Tirants filetés en bout avec écrou et contre-écrou maintenant l'écartement des pieds. - (I 3 ) Butée arrière du plancher. - (I4) Espace libre minimum assurant le passage des fèces quand (13) vient buter dans la porte arrière, le plancher étant alors développé au maximum. - (I5) 7 barreaux amovibles permettant de supporter l'animal et de laisser passer ses crottes. $-25 \mathrm{~mm}$ entre axe, section des barreaux : Io $\mathrm{mm}$. - (16) Bac à fèces avec porte et charnière en haut s'ouvrant vers l'arrière. - (I7) Entonnoir à urine. - (18) Grille inclinée séparatrice de crottes amovible et fixée à l'aide de papillons sur le châssis du plancher. - (19) Cirille à trous fins de $3 \mathrm{~mm}$ (en traits croisés). - (20) Auge mobile.

\section{4. - Séparation fèces-urines}

\section{a) Description des différentes parties:}

Le plancher (Io) qui porte l'animal est constitué d'une tôle métallique perforée de trous de $8 \mathrm{~mm}$ de diamètre disposés en quinconce. La rigidité est obtenue par un cadre métallique qui coulisse d'avant en arrière sur des glissières (ך). 
Le plancher, ainsi constitué, peut être retiré de l'avant, comme de l'arrière de la cage.

La partie arrière du cadre métallique est libre.

Dans le cas d'utilisation de femelles cet espace libre permet de mettre en place la grille inclinée séparatrice de fèces (I8) façonnnée en tôle perforés de trous de $6 \mathrm{~mm}$ de diamètre.

Cette grille n'étant pas nécessaire avec les mâles, la partie libre du cadre peut être alors occupée par des barreaux amovibles supportant l'arrière de l'animal, mais laissant passer les fèces dans le "bac " (I6).

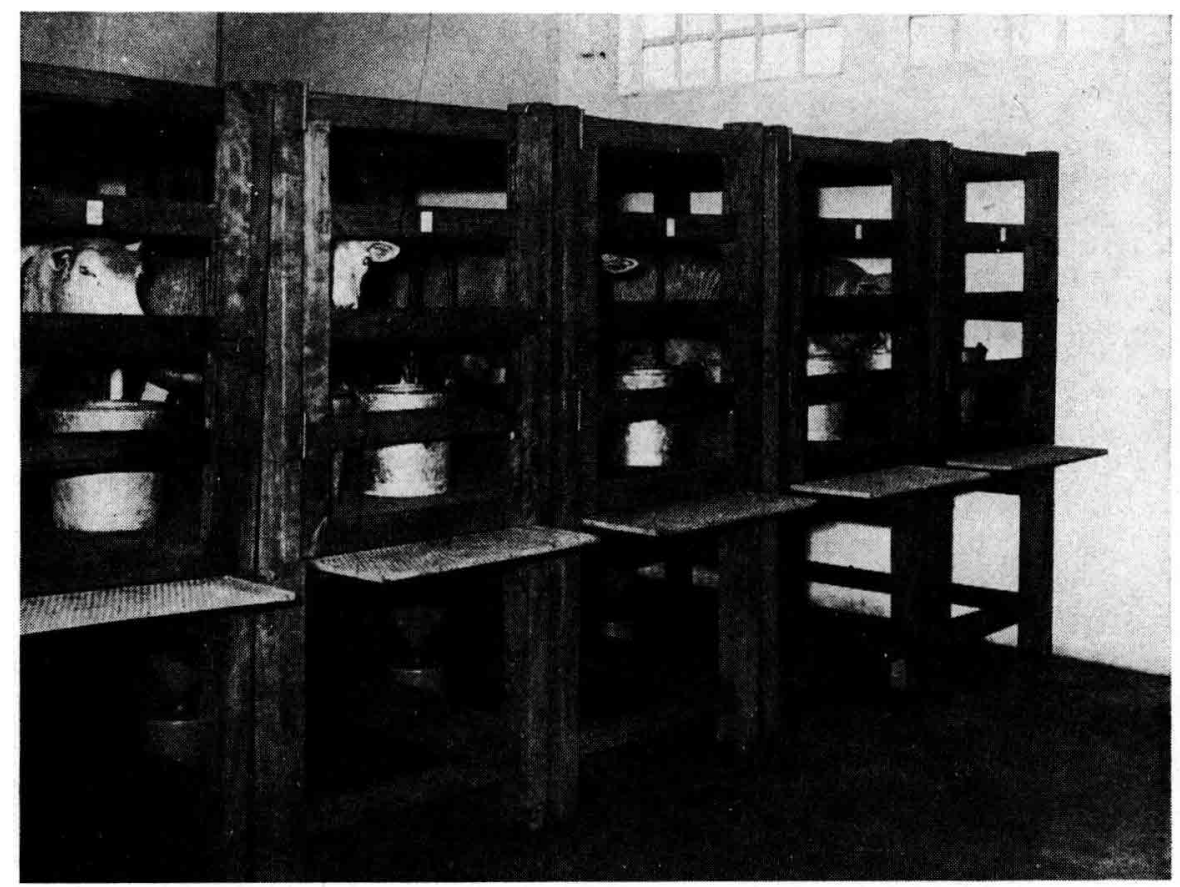

FIG. 4. Vue avant.

$I_{4}$ e bac à fèces, en tôle, est ouvert vers 1'arrière par une porte s'ouvrant vers le haut, ce qui permet de faire la récolte sans déranger ni 1'animal ni le bac lui-même.

I'urine est récupérée par un entonnoir en tôle ( $\mathrm{I} 7$ ), reposant sur la même glissière (9) que le bac à fèces.

Une protection supplémentaire est apportée par une grille en tôle perforée de petits trous de $3 \mathrm{~mm}$ de diamètre (I9), qui couvre la totalité de l'entonnoir et retient les débris (poils...) passant à travers le plancher.

L'ensemble, bac à fèces et entonnoir à urine, peut être retiré sans déplacer le plancher ni l'animal. 


\section{b) Réglage. Fonctionnement.}

Dans le cas le plus simple, utilisation des mâles, le seul réglage est celui du bac à fèces et de l'entonnoir à urine par rappott au plancher qui doit être plus ou moins glissé vers l'avant, en fonction de la longueur de l'animal.

Pour les femelles, le plancher doit être arancé au maximum, jusqu'à

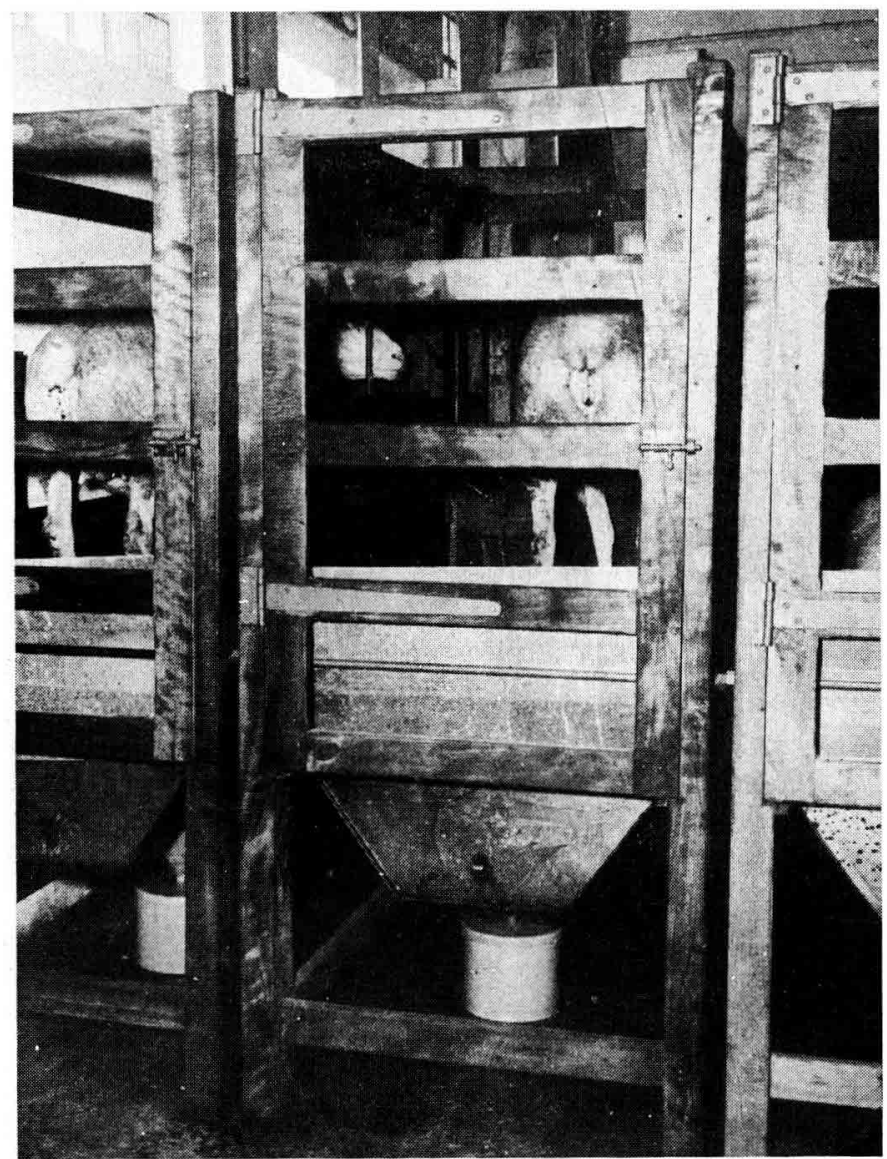

FIs. 5. - Vhe arritre.

ce que la saillie de la grille séparatrice vienne tout près du pâturon des brebis en station debout.

I,es animaux défèquent soit conchés, soit debout. Dans les deux positions les fèces tombent sur la grille inclinée et roulent dans le bac.

Il est extrêmement rare que les animaux urinent en étant couchés, de plus, les femelles se voussent pour la mixion. Par conséquent, le jet d'urine très court vient frapper la grille séparatrice où les éclaboussures 
sont réduites au minimum sur ce plan incliné ; enfin le ruissellement est presque annulé par la disposition des trous en quinconce.

Malgré tout, on effectue le réglage de manière à ce que l'extrémité inférieure de la grille séparatrice soit placée au-dessus de l'entonnoir à

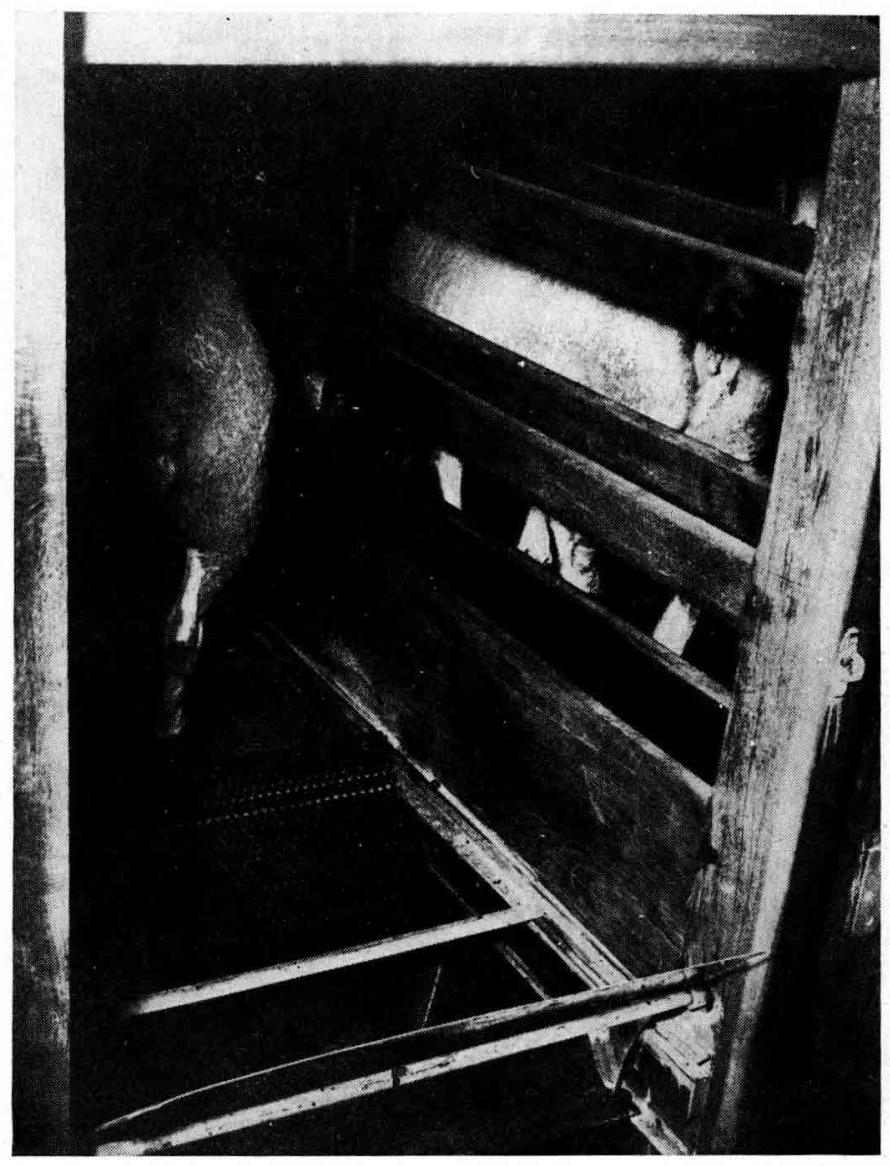

FIr. 6. - Ditail arriere.

urine, l'intervalle libre (I à $2 \mathrm{~cm}$ ) étant couvert par la grille de protection (I9). I'urine s'égoutte alors dans l'entonnoir et les crottes roulent dans le bac.

$$
* *
$$

Ce modèle de cage de digestibilité, pouvant servir pour moutons, brebis ou veaux, a été utilisé avec satisfaction pendant I an avec des femelles. La séparation des urines et fèces, obtenue par grille inclinée, est bonne et permet des récoltes sans contanination. I,es animaux peuvent $y$ être maintenus pendant des mois. Avec quelques modifications de détail, ce type de cage peut être adapté à de nombreux besoins. 


\section{RÉFÉRENCES BIBLIOGRAPHIQUES}

BASSET (E. G.). - Apparatus for continuous collection of urine from grazing ewe. N. Z. J. of Sc. and Techn. Sect. A, 34, no I, 76-8I, I952.

Bratzler (J. W.). - A metabolism crate for use with sheep. J. of Animal Sci., 10, 593-60I, I95I.

BRIGGS (M. M.), GALLUP (W. D,). - Metabolism stalls for wethers and steers. $J$. of Animal Sci., 8, 479-482, I949.

Burkitr (W. H.). - Apparent digestibility by lambs of grass hay supplemented with rape seed oil meal or linseed meal. Montana A gr. Exp. Sta. Circ., I93, I95I.

DICK (A. T.), MULES (M. W.). - Equipment for the clean collection of twenty four hours sample of urine and feces from sheep. Aust. J. of Agr. Research, 5, 345-347, I 954 .

Forbes (E. B.), Bratzler (J. W.), Black (A.), Braman (W. W.). -- The digestibility of rations by cattle and sheep. Pennsylvania Agr. Exp. Sta. Bull., 339, I937.

Hodgson (R. E.), Knott (J. C.), Graves (R. R.), Murer (H. K.). - Effect of temperature of artificial draying on digestibility and availability of nutrients of artificial draying on digestibility and availability of nutrients in pasture herbage. J. of $A g r$. Research., 50, I49-I64, I935.

Nelison (A. B.), Tillmann (A. D.), Gallup (W. O.), McVicar (R.). - Modified metabolism stall for steers. J. of Animal Sci., 13, 504-5Io, r954.

Raymond (W. F.), Harris (C. E.), Harker (V. G.). - Studies on the digestibility of herbage. I. Technique of measurement of digestibility and some observations on factors affecting the accuracy of digestibility data. J. of the Brit. Grass. Soc., 8, 30I-3I4, I953.

SotorA (J.). - Relation of maturity to the nutritive value of first, second and third cuttings of irrigated alfalfa. J. of $A g r$. Research., 35, 36I384 , I927.

Le Directeur-Gérant : B. LACLAVIÈRE.

I.N.R.A

B/BL IOTHEQUE UO 35906primerie BussiżkE, Saint-Amand (Cher), France. - 18-3-1958

DOHAINE DE CROUELLE Dépót légal: 1er Trimestre 1958 No d'impression: 776

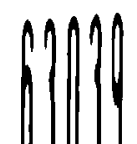

